\title{
ENTOURAGES, COVERS AND LOCALIC GROUPS
}

\author{
JORGE PICADO AND ALEŠ PULTR
}

\begin{abstract}
Due to the nature of product in the category of locales, the entourage uniformities in the point-free context only mimic the classical Weil approach while the cover (Tukey type) ones can be viewed as an immediate extension. Nevertheless the resulting categories are concretely isomorphic. We present a transparent construction of this isomorphism, and apply it to the natural uniformities of localic groups. In particular we show that localic group homomorphisms are uniform, thus providing natural forgetful functors from the category of localic groups into any of the two categories of uniform locales.
\end{abstract}

\section{INTRODUCTION}

In the classical spaces, a uniformity on $X$ is approached, basically, by two different (but equivalent) ways:

- one can take systems of special covers of $X$ - the Tukey mode (see e.g. $[23,10]$ ),

- or one can consider systems of special "neighborhoods of the diagonal" (entourages) in the product $X \times X$ - the Weil mode $([24])$.

Both can be extended to the point-free spaces (locales). Thus we have an extensive literature about the cover uniformities, starting with the pioneering Isbell's [11] (further see e.g. [1, 2, 3, 9, 21, 22], etc.). On the other hand, the entourage and kindred types of uniformities were thoroughly studied e.g. in $[18,19,20]$ and from another technical standpoint e.g. in $[7,8]$ (for a discussion of the relation with the entourage technique see $[6])$.

2000 Mathematics Subject Classification. 06D22, 18B30, 18B99, 18D35, 22A05, $54 \mathrm{E} 15$.

Key words and phrases. Frame, locale, frame coproduct, localic product, cover, entourage, cover and entourage uniformity, localic group.

Thanks go to the Centre for Mathematics of the University of Coimbra (CMUC/FCT), to the project MTM2009-12872-C02-02 of the Ministry of Science and Innovation of Spain and to the projects 1M0545 and MSM 0021620838 of the Ministry of Education of the Czech Republic. 
Comparing the two extensions we see a fundamental difference. The cover one is immediate in the sense that a classical uniformity on a space $X$ coincides with the point-free one on the frame of open sets $\Omega(X)$. The entourage uniformity, however, happens in the product $X \times X$; now the category of (completely regular) locales extending that of (completely regular) spaces is much bigger, and the coproduct in this bigger category considerably differs (the embedding CRegSp $\rightarrow$ CRegLoc does not preserve products; the classical entourage uniformity is something that happens in $\Omega(X \times X)$ while the point-free extension applied for $X$ happens in $\Omega(X) \times \Omega(X)$ in Loc which is generally something else). Yet, the two approaches are, again, equivalent (which is a somewhat deeper fact than what meets the eye, and should come, in a way, as a bit of surprise).

As we have already said, there is abundant literature on both the cover and the entourage uniformities. The relation between the two, however, has been somewhat neglected. The equivalence was proved by the first author in his Thesis ([18]; cf. [19]) using a certain technical detour; but this seems to be about all. Thus, one does not have, to our knowledge, a direct proof of the equivalence in the standard journal literature; one of the aims of this paper is to fill in this gap.

Further, we concentrate on the uniform structure of localic groups (analogues of topological groups in the point-free context). In the original article about this subject ([12]) it was shown that similarly like in the classical case one has natural cover uniformities induced by the group structure. In fact one has equally (if not even more) natural entourage ones $([20])$. We describe them and show their equivalence with the cover uniformities; while doing this we also discuss the semigroup of open parts of a localic group (which has not yet been presented in this detail). As an application we present an extremely simple proof of the fact that the localic group homomorphisms are uniform; it should be noted that this fact has so far, to our knowledge, not been proved in the literature by the cover methods, and even remaking our simple entourage proof to a cover one by translation seems to be rather complex. We see it as another corroboration of the usefulness of the entourage approach.

Only basic knowledge of classical topology is assumed. The necessary definitions and facts concerning frames (locales) are presented in Preliminaries below. 


\section{Preliminaries}

1.1. Recall that a frame is a complete lattice satisfying the distributive law

$$
(\bigvee A) \wedge b=\bigvee\{a \wedge b \mid a \in A\}
$$

for all subsets $A \subseteq L$ and all $b \in L$. A frame homomorphism $h: L \rightarrow M$ preserves all joins (including the void one, the bottom 0 ) and all finite meets (including the top 1 ). The resulting category will be denoted by

\section{Frm.}

A typical frame is the lattice $\Omega(X)$ of all open sets of a topological space $X$; if $f: X \rightarrow Y$ is a continuous map then $\Omega(f)=(U \mapsto$ $\left.f^{-1}[U]\right): \Omega(Y) \rightarrow \Omega(X)$ is a frame homomorphism. Thus one has a contravariant functor $\Omega:$ Top $\rightarrow$ Frm (where Top is the category of topological spaces). Setting

$$
\text { Loc }=\text { Frm }^{\text {op }}
$$

one obtains the category of locales; then, $\Omega$ becomes a contravariant functor Top $\rightarrow$ Loc.

1.2. Restricted to the subcategory Sob of sober spaces, $\Omega$ is a full embedding. In this paper we will be interested in the category of completely regular spaces resp. locales (notation CRegSp resp. CRegLoc) and, as usual in the point-free context, the spaces will be always $T_{0}$. Thus, our spaces will be always Hausdorff and hence in particular sober, We will write

$$
\mathfrak{O}: \text { CRegSp } \rightarrow \text { CRegLoc }
$$

for the full embedding restriction of the covariant $\Omega$ and we will think of CRegLoc as of a natural extension of CRegSp.

1.3. For more about frames and locales see e.g. [13, 22] (technically, we will mostly work with the comfortable frame techniques). Since, however, the product of locales (coproduct of frames) will be crucial, let us present a simple construction of the coproduct of two frames (see e.g. $[13,22]$; it should be noted that the first construction of frame coproducts appeared in [5]). The subcategory of completely regular frames is coreflective in Frm; hence the construction applies for our special case as well.

First take the Cartesian product $L \times M$ as a poset and $\mathfrak{D}(L \times M)=$ $\{U \subseteq L \times M \mid \downarrow U=U \neq \emptyset\}$ (where $\downarrow U=\{(x, y) \mid(x, y) \leq(a, b) \in U\}$, as usual). Call a $U \in \mathfrak{D}(L \times M)$ saturated if 
(1) for any subset $A \subseteq L$ and any $b \in M$, if $A \times\{b\} \subseteq U$ then $(\bigvee A, b) \in U$, and

(2) for any $a \in L$ and any subset $B \subseteq M$, if $\{a\} \times B \subseteq U$ then $(a, \bigvee B) \in U$.

The set $A$ resp. $B$ can be void; hence, in particular, each saturated set contains as a subset

$$
\mathrm{n}=\{(0, b),(a, 0) \mid a \in L, b \in M\} .
$$

It is easy to check that for each $(a, b) \in L \times M$,

$$
a \oplus b=\downarrow(a, b) \cup \mathrm{n} \text { is saturated. }
$$

To finish the construction take

$$
L \oplus M=\{U \in \mathfrak{D}(L \times M) \mid U \text { saturated }\}
$$

with the coproduct injections

$$
\iota_{L}=(a \mapsto a \oplus 1): L \rightarrow L \oplus M, \quad \iota_{M}=(b \mapsto 1 \oplus b): M \rightarrow L \oplus M
$$

Note that we have

(1.3.1) for each saturated $U$,

$$
U=\bigvee\{a \oplus b \mid(a, b) \in U\}=\bigcup\{a \oplus b \mid(a, b) \in U\}, \quad \text { and }
$$

(1.3.2) if $a \oplus b \leq c \oplus d$ and $b \neq 0$ then $a \leq c$.

Convention. Using the symbol $L \times M$ when speaking of $L \oplus M$ as a product in the category Loc would probably obscure the matter. Therefore, we will keep the notation $L \oplus M$ also in Loc. We only have to keep in mind that then the injections $\iota$ become projections $\iota_{L}, \iota_{M}: L \oplus M \rightarrow L, M$.

1.4. The category CRegLoc is much bigger than the category CRegSp (more precisely, the image of CRegSp under $\mathfrak{O}$ ) and hence it is no surprise that the products in CRegLoc considerably differ from those in CRegSp. That is, the embedding $\Omega$ resp. $\mathfrak{O}$ does not generally preserve products (it is a left adjoint, not a right one). One does have $\mathfrak{O}(X \times Y) \cong \mathfrak{O}(X) \oplus \mathfrak{O}(Y)$ in some important special cases, but by far not in the generality of all the completely regular spaces.

\section{Cover (Tukey) Uniformities}

2.1. A cover of a frame $L$ is a subset $U \subseteq L$ such that $\bigvee U=1$. A cover $U$ refines (or is a refinement of) a cover $V$ (written, $U \leq V$ ) if

$$
\forall u \in U \exists v \in V \quad \text { such that } u \leq v .
$$


For covers $U, V$ we have the largest common refinement

$$
U \wedge V=\{u \wedge v \mid u \in U, v \in V\} .
$$

If $U \subseteq L$ is a cover and $a \in L$ we set

$$
U a=\bigvee\{u \in U \mid u \wedge a \neq 0\}
$$

and for covers $U, V$ define

$$
U V=\{U v \mid v \in V\} .
$$

Note that if $U$ is a cover of $L$ and if $h: L \rightarrow M$ is a frame homomorphism then

$$
h[U] h(a) \leq h(U a)
$$

(if $h(u) \wedge h(a) \neq 0$ then $u \wedge a \neq 0$ and hence $h(u) \leq h(U a)$ for $u \in U$ ).

Finally, for a set of covers $\mathcal{U}$ define a relation

$$
b \triangleleft_{\mathcal{U}} a \equiv_{\mathrm{df}} \text { there is a } U \in \mathcal{U} \text { such that } U b \leq a .
$$

$\mathcal{U}$ is said to be admissible if

$$
\forall a \in L, \quad a=\bigvee\left\{b \mid b \triangleleft_{\mathcal{U}} a\right\}
$$

2.2. A cover-uniformity (briefly, c-uniformity) on a frame $L$ is an admissible non-empty system of covers $\mathcal{U}$ such that

(U1) if $U \in \mathcal{U}$ and $U \leq V$ then $V \in \mathcal{U}$,

(U2) if $U, V \in \mathcal{U}$ then $U \wedge V \in \mathcal{U}$,

(U3) for every $U \in \mathcal{U}$ there is a $V \in \mathcal{U}$ such that $V V \leq U$.

Often one works with a basis of a uniformity $\mathcal{B}$, a system satisfying just (U2) and (U3). Then one obtains a uniformity taking all the $U \geq V$ with $V \in \mathcal{B}$.

A $c$-uniform frame resp. c-uniform locale is a pair $(L, \mathcal{U})$ where $\mathcal{U}$ is a c-uniformity on $L$. A frame homomorphism $h: L \rightarrow M$ is a uniform homomorphism $(L, \mathcal{U}) \rightarrow(M, \mathcal{V})$ if

$$
\forall U \in \mathcal{U}, \quad h[U] \in \mathcal{V}
$$

(if $\mathcal{U}, \mathcal{V}$ are bases of uniformities this condition is replaced by

$$
\forall U \in \mathcal{U} \exists V \in \mathcal{V} \quad \text { such that } h[U] \geq V .)
$$

The resulting category, in the localic setting, will be denoted by

\section{Uni $_{\text {CLoc. }}$ Lot}

2.3. If $U a \leq b$ then $a \prec b$ (since $1=\bigvee U=U a \bigvee \bigvee\{x \in U \mid x \wedge a=$ $0\}$ and $\left.\bigvee\{x \in U \mid x \wedge a=0\} \leq a^{*}\right)$. Furthermore, if $\mathcal{U}$ is a uniformity then the relation $\triangleleft_{\mathcal{U}}$ interpolates, that is, if $a \triangleleft_{\mathcal{U}} b$ then there is a $c$ such that $a \triangleleft_{\mathcal{U}} c \triangleleft_{\mathcal{U}} b$ (if $U a \leq b$ take a $V$ from (U3) and set 
$c=V a$; use the easy fact that $U(V x) \leq(U V) x$ to obtain $V c \leq b)$. Thus, $a \triangleleft_{\mathcal{U}} b \Rightarrow a \prec b$ and hence each $L$ admitting a uniformity is completely regular and we have the forgetful functor

$$
((L, \mathcal{U}) \mapsto L): \text { Uni }_{\mathbf{C}} \text { Loc } \rightarrow \text { CRegLoc }
$$

2.4. The classical Tukey uniformities. A cover uniformity on a space $X$ in the classical setting is nothing else but a c-uniformity on $\mathfrak{O}(X)$ and a continuous map $f: X \rightarrow Y$ is a uniformly continuous $\operatorname{map}(X, \mathcal{U}) \rightarrow(Y, \mathcal{V})$ precisely when $\Omega(f)$ is a uniform homomorphism. Thus, if we denote by $\mathbf{U n i}_{\mathbf{C}} \mathbf{S p}$ the category of classical (cover) uniform spaces, we have a natural full embedding

$$
\mathfrak{O}_{\mathrm{C}}: \mathrm{Uni}_{\mathrm{C}} \mathrm{Sp} \rightarrow \mathrm{Uni}_{\mathrm{C}} \mathrm{Loc}
$$

carried by $\mathfrak{O}$.

\section{Entourage (Weil) Uniformities.}

3.1. An entourage in $L$ is an element $E \in L \oplus L$ such that

$$
\{u \mid u \oplus u \leq E\}
$$

is a cover of $L$.

For entourages $E, F$ of $L$ set

$$
\begin{aligned}
E \circ F & =\bigvee\{a \oplus c \mid \exists b \neq 0, \quad a \oplus b \leq E \text { and } b \oplus c \leq F\}= \\
& =\bigvee\{a \oplus c \mid \exists b \neq 0, \quad(a, b) \in E \text { and }(b, c) \in F\} .
\end{aligned}
$$

(Caution: unions of saturated sets are not necessarily saturated and the join above is typically bigger than the corresponding union.)

Further, for an entourage $E$ set

$$
E^{-1}=\{(a, b) \mid(b, a) \in E\}
$$

(which is obviously an entourage again).

If $E$ is an entourage (resp. $\mathcal{E}$ a set of entourages) write

$b \triangleleft_{E} a$ if $E \circ(b \oplus b) \leq a \oplus a, \quad$ and $\quad b \triangleleft_{\mathcal{E}} a$ if $\exists E \in \mathcal{E}, b \triangleleft_{E} a$.

A set of entourages $\mathcal{E}$ is said to be admissible if

$$
\forall a \in L, \quad a=\bigvee\left\{b \mid b \triangleleft_{\mathcal{E}} a\right\} .
$$

An entourage-uniformity (briefly, e-uniformity) on a frame $L$ is an admissible set of entourages $\mathcal{E}$ such that

(E1) if $E \in \mathcal{E}$ and $E \leq F$ then $F \in \mathcal{E}$, 
(E2) if $E, F \in \mathcal{E}$ then $E \cap F \in \mathcal{E}$,

(E3) if $E \in \mathcal{E}$ then $E^{-1}$ is in $\mathcal{E}$, and

(E4) for every $E \in \mathcal{E}$ there is an $F \in \mathcal{E}$ such that $F \circ F \leq E$.

Note that obviously intersections of saturated elements are saturated; hence the $E \cap F$ in (E2) makes sense.

Similarly as above, a basis of a uniformity $\mathcal{B}$ is a system satisfying just (E2) and (E3) and (E4) and a uniformity is obtained by taking all the $F \geq E$ with $E \in \mathcal{B}$.

3.2. An e-uniform frame resp. e-uniform locale is a pair $(L, \mathcal{E})$ where $\mathcal{E}$ is an e-uniformity on $L$. A frame homomorphism $h: L \rightarrow M$ is a uniform homomorphism $(L, \mathcal{E}) \rightarrow(M, \mathcal{F})$ if

$$
\forall E \in \mathcal{E}, \quad(h \oplus h)(E) \in \mathcal{F}
$$

(where $h \oplus h$ is the frame homomorphism $L \oplus L \rightarrow M \oplus M$ defined by $(h \oplus h) \iota_{i}=\iota_{i} h$ for $\left.i=1,2\right)$. If $\mathcal{E}, \mathcal{F}$ are bases of uniformities this condition is replaced by

$$
\forall E \in \mathcal{E} \exists F \in \mathcal{F} \quad \text { such that } \quad(h \oplus h)(E) \geq F .
$$

The resulting category, in the localic setting, will be denoted by

\section{Uni $_{\mathrm{E}}$ Loc.}

3.3. It is easy to prove that, again, $a \triangleleft_{\mathcal{E}} b$ implies $a \prec b$ so that if $L$ admits an e-uniformity it is completely regular and hence we have the forgetful functor $((L, \mathcal{E}) \mapsto L): \mathbf{U n i}_{\mathbf{E}}$ Loc $\rightarrow$ CRegSp. But it is not quite as straightforward as in the cover case and we will get it for free in the next section anyway. Therefore we need not go into it now.

3.4. A more suspect thing is the relation of our entourage uniformity with the classical one. Recall that a Weil uniformity on a topological space $X$ is a non-empty system $\mathcal{E}$ of open subsets of the product $X \times X$, each containing the diagonal $\Delta=\{(x, x) \mid x \in X\}$, such that

(E'1) if $U \in \mathcal{E}$ and $U \subseteq V$ then $V \in \mathcal{E}$,

(E'2) if $U, V \in \mathcal{E}$ then $U \cap V \in \mathcal{E}$,

(E'3) if $U \in \mathcal{E}$ then $U^{-1}$ is in $\mathcal{E}$, and

(E'4) for every $U \in \mathcal{E}$ there is an $V \in \mathcal{E}$ such that $V \circ V \leq E$

(where $\circ$ indicates the standard composition of binary relations, and $\left.U^{-1}=\{(x, y) \mid(y, x) \in U\}\right)$, such that

(Adm) for each $x \in X$, the system $\{U x=\{y \mid(x, y) \in U\} \mid U \in \mathcal{E}\}$ is the basis of neighbourhoods in the original topology. 
Now we see that the condition in the definition of entourage that $\{u \mid u \oplus u \leq E\}$ is a cover stands for $U$ containing the diagonal, the admissibility makes for the (Adm) and the (Ei)'s are analogues of the classical (E'i)'s so that the entourage uniformity above seems to be a natural extension of the classical one. But, even if we believe the statement in 3.3 , the fact that the functor $\mathfrak{O}$ really carries a full embedding $\mathfrak{O}_{\mathbf{E}}: \mathbf{U n i}_{\mathbf{E}} \mathbf{S p} \rightarrow \mathbf{U n i}_{\mathbf{E}} \mathbf{L o c}$ (again) should come at least as a minor pleasant surprise: for a space $X$ the classical Weil uniformity happens in $\Omega(X \times X)$ while the entourage uniformity from 3.2 is defined in $\Omega(X) \oplus \Omega(X)$ which is by far not the same.

\section{Translations}

4.1.1. Lemma. Let $U$ be a cover of $L$, let $x \oplus y \leq \bigvee\{u \oplus u \mid u \in U\}$ and let $y \neq 0$. Then $x \leq U y$.

Proof. We have

$$
\begin{aligned}
x \oplus y & =\bigvee\{(u \wedge x) \oplus(u \wedge y) \mid u \in U\}= \\
& =\bigvee\{(u \wedge x) \oplus(u \wedge y) \mid u \in U, u \wedge y \neq 0\} \leq(U y \wedge x) \oplus y .
\end{aligned}
$$

Thus, if $y \neq 0, x \leq x \wedge U y$, and finally $x \leq U y$.

4.1.2. Lemma. If $E$ is an entourage then $E \leq E \circ E$.

Proof. Let $a \oplus b \leq E$ and $b \neq 0$. We have

$$
b=\bigvee\{b \wedge u \mid u \oplus u \leq E\}=\bigvee\{b \wedge u \mid u \oplus u \leq E, u \wedge b \neq 0\} .
$$

Now we have, for $b \wedge u \neq 0, a \oplus(b \wedge u) \leq E$ and $(b \wedge u) \oplus(b \wedge u) \leq E$, hence $a \oplus(b \wedge u) \leq E \circ E$ and hence $a \oplus b=a \oplus \bigvee\{b \wedge u \mid u \oplus u \leq$ $E\}=\bigvee\{a \oplus(b \wedge u) \mid u \oplus u \leq E\} \leq E \circ E$.

4.2. For an entourage $E$ define

$$
\widetilde{E}=\bigvee\{u \oplus u \mid u \oplus u \leq E\}
$$

Note that trivially $\widetilde{E} \leq E$.

4.2.1. Lemma. Let $F$ be a symmetric entourage and let $F \circ F \leq E$. Then for each $0 \neq a \oplus b \leq F$ we have $(a \vee b) \oplus(a \vee b) \leq E$.

Consequently, if $\mathcal{E}$ is a uniformity then $\widetilde{E} \in \mathcal{E}$ for each $E \in \mathcal{E}$.

Proof. Let $a \oplus b \leq F$, so that also $b \oplus a \leq F$ and hence

$$
a \oplus a, b \oplus b \leq F \circ F \leq E
$$


by 4.1 .2 also

$$
a \oplus b, b \oplus a \leq F \circ F \leq E .
$$

Thus $a \oplus(a \vee b) \leq E, b \oplus(a \vee b) \leq E$ and finally $(a \vee b) \oplus(a \vee b) \leq E$ and we conclude that $a \oplus b \leq(a \vee b) \oplus(a \vee b) \leq \widetilde{E}$.

4.3. Translations. For a cover $U$ define an entourage $E_{U}$, and for an entourage $E$ define a cover $U_{E}$ by setting

$$
E_{U}=\bigvee\{x \oplus x \mid x \in U\} \quad \text { and } \quad U_{E}=\{x \mid x \oplus x \leq E\} .
$$

4.3.1. Lemma. (a) $U \leq U_{E_{U}} \leq U U$.

(b) $\widetilde{E}=E_{U_{E}} \leq E$.

Proof. (a) If $x \oplus x \leq E_{U}$ then for any $u_{0} \in U$ such that $u_{0} \wedge x \neq 0$ we have by 4.1 .1

$$
x \leq U\left(u_{0} \wedge x\right) \leq U u_{0}
$$

Thus,

$$
U_{E_{U}}=\left\{x \mid x \oplus x \leq E_{U}\right\} \leq U U .
$$

On the other hand, trivially, $U \leq U_{E_{U}}$.

(b) follows immediately from the definitions.

4.3.2. Lemma. (a) $b \triangleleft_{E} a \Rightarrow U_{E} b \leq a$.

(b) $U b \leq a \Rightarrow b \triangleleft_{E_{U}} a$.

Proof. (a) Let $u \in U_{E}$ and $u \wedge b \neq 0$. Then $u \oplus(u \wedge b) \leq E$ and $(u \wedge b) \oplus b \leq b \oplus b$, and hence $u \oplus b \leq E \circ(b \oplus b) \leq a \oplus a$; thus, as $b \neq 0, u \leq a$ and we conclude that $U_{E} b \leq a$.

(b) Let $U b \leq a$ and let $x \oplus y \leq E_{U}$ and $y \oplus z \leq b \oplus b$ for some $y \neq 0$. Then $x \oplus y \leq \bigvee\{u \oplus u \mid u \in U\}$ and by 4.1.1, $x \leq U y$. Thus, $x \oplus z \leq U y \oplus b \leq U b \oplus b \leq a \oplus a$.

4.4. For an entourage-uniformity $\mathcal{E}$ set $\mathcal{U}_{\mathcal{E}}=\left\{V \mid V \geq U_{E}, E \in \mathcal{E}\right\}$ and for a cover-uniformity $\mathcal{U}$ define $\mathcal{E}_{\mathcal{U}}=\left\{F \mid F \geq E_{U}, U \in \mathcal{U}\right\}$.

4.4.1. Proposition. If $\mathcal{E}$ is an e-uniformity then $\mathcal{U}_{\mathcal{E}}$ is a $c$ uniformity, and if $\mathcal{U}$ is a c-uniformity then $\mathcal{E}_{\mathcal{U}}$ is an e-uniformity. We have $\mathcal{E}_{\mathcal{U}_{\mathcal{E}}}=\mathcal{E}$ and $\mathcal{U}_{\mathcal{E}_{\mathcal{U}}}=\mathcal{U}$

Proof. By 4.3.2, $\triangleleft_{\mathcal{U}}=\triangleleft_{\mathcal{E}_{\mathcal{U}}}$ (and $\triangleleft_{\mathcal{E}}=\triangleleft_{\mathcal{U}_{\mathcal{E}}}$ ). Thus, if any of the associated uniformities is admissible then the other one is admissible as well.

The formulas $\mathcal{E}_{\mathcal{U}_{\mathcal{E}}}=\mathcal{E}$ and $\mathcal{U}_{\mathcal{E}_{\mathcal{U}}}=\mathcal{U}$ follow from 4.3.1.

Thus, it remains to be proved that the systems $\mathcal{U}_{\mathcal{E}}$ and $\mathcal{E}_{\mathcal{U}}$ are cresp. e-uniformities. We will prove (U3) and (E4), the other facts are straightforward. 
To prove (U3) we will show that if $F$ is symmetric and $(F \circ F) \circ(F \circ$ $F) \leq E$ then $U_{F} U_{F} \leq U_{E}$. Set $F_{1}=F \circ F$. Fix a $u \in U_{F}$ and take an arbitrary $v \in U_{F}$ such that $u \wedge v \neq 0$. Then because of $u \wedge v \neq 0$, $v \oplus u \leq F_{1}=F \circ F$ and since $F_{1}$ is saturated,

$$
U_{F} u \oplus u=(\bigvee\{v \mid v \wedge u \neq 0\}) \oplus u \leq F_{1} .
$$

Since $F$ is symmetric, $F_{1}$ is symmetric as well and hence by 4.2.1, $U_{F} u \oplus U_{F} u \leq F_{1} \circ F_{1} \leq E$, and $U_{F} u \in U_{E}$.

To prove (E4) we will show that $E_{U} \circ E_{U} \leq E_{U U}$. Take an $x \oplus z \leq$ $E_{U} \circ E_{U}$; hence there is a $y \neq 0$ such that $x \oplus y \leq E_{U}$ and $y \oplus z \leq E_{U}$. Choose a $u \in U$ such that $u \wedge y \neq 0$. Since $x \oplus(u \wedge y) \leq E_{U}$ and $(u \wedge y) \oplus z \leq E_{U}$ we obtain from 4.1.1 that $x \oplus z \leq U u \oplus \overline{U u} \leq E_{U U}$.

4.4.2. Theorem. The categories $\mathbf{U} \mathbf{n i}_{\mathbf{E}} \mathbf{L o c}$ and $\mathbf{U n i}_{\mathbf{C}} \mathbf{L o c}$ are concretely isomorphic with the isomorphism functors $\mathbb{C}: \mathbf{U n i}_{\mathbf{E}} \mathbf{L o c} \rightarrow$ $\mathbf{U n i}_{\mathbf{C}} \mathbf{L o c}$ and $\mathbb{E}: \mathbf{U n i}_{\mathbf{C}} \mathbf{L o c} \rightarrow \mathbf{U n i}_{\mathbf{E}}$ Loc carried by the identity.

Proof. Set $\mathbb{C}(L, \mathcal{E})=\left(L, \mathcal{U}_{\mathcal{E}}\right)$ and $\mathbb{E}(L, \mathcal{U})=\left(L, \mathcal{E}_{\mathcal{U}}\right)$. After 4.4 .1 it remains to be proved that the concepts of uniform homomorphisms in the associated c- and e-uniformities coincide.

I. Suppose that for each $E \in \mathcal{E}$ there exists an $F \in \mathcal{F}$ such that

$$
(h \oplus h)(E) \geq F .
$$

Take $U \in \mathcal{U}$ and a $V \in \mathcal{U}$ such that $V V \leq U$. By (*) (and 3.1) there exists in particular a $W \in \mathcal{V}$ such that

$$
(h \oplus h)\left(E_{V}\right) \geq E_{W} .
$$

Take a $w \in W$. Then $w \oplus w \leq \bigvee\{h(v) \oplus h(v) \mid v \in V\}$ and hence, by 4.4.1, if we take a $v_{0} \in V$ such that $y=w \wedge h\left(v_{0}\right) \neq 0$ we obtain that $w \leq h[V] y \leq h[V] h\left(v_{0}\right) \leq h\left(V v_{0}\right) \leq h(u)$ for some $u \in U$. Thus, $W \leq h[U]$.

II. Let for each $U \in \mathcal{U}$ there be a $V \in \mathcal{V}$ such that

$$
h[U] \geq V .
$$

Consider an $E \in \mathcal{E}$. There is an $F \in \mathcal{F}$ such that $h\left[U_{E}\right] \geq U_{F}$. Let $v \oplus v \leq F$. Then $v \in U_{F}$ and hence there is a $u \in U_{E}$ such that $v \leq h(u)$. Then

$$
v \oplus v \leq h(u) \oplus h(u) \leq(h \oplus h)(u \oplus u) \leq(h \oplus h)(E)
$$

and hence $(h \oplus h)(E) \geq \widetilde{F}$. Recall 4.2 . 
4.5. From 4.3 .2 and the ensuing equalities $\triangleleft_{\mathcal{U}}=\triangleleft_{\mathcal{E}_{\mathcal{U}}}$ and $\triangleleft_{\mathcal{E}}=\triangleleft_{\mathcal{U}_{\mathcal{E}}}$, and from 2.3 we see that the underlying locale of an e-uniform locale is completely regular (as indicated in 3.3). Now consider the diagram

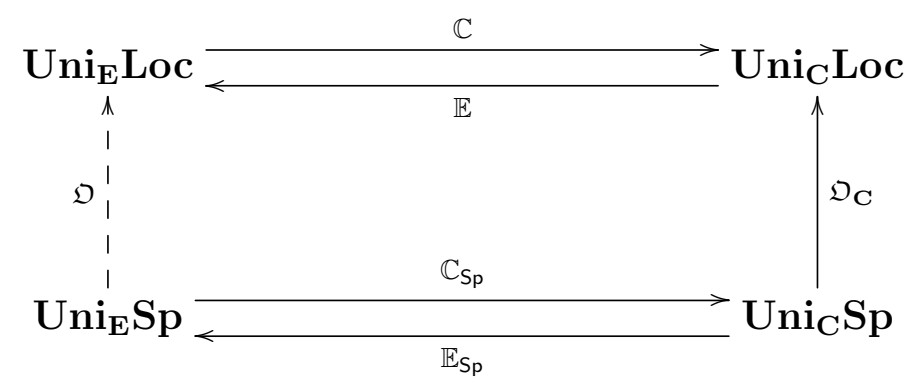

where $\mathbb{C}_{S p}$ and $\mathbb{E}_{S p}$ are the concrete isomorphism functors representing the standard classical equivalence of Tukey and Weil uniformities. We have a full embedding

$$
\mathfrak{O}_{\mathrm{E}}: \mathrm{Uni}_{\mathrm{E}} \mathrm{Sp} \rightarrow \mathrm{Uni}_{\mathrm{E}} \mathrm{Loc}
$$

carried by $\mathfrak{O}$ defined by $\mathfrak{O}_{\mathbf{E}}=\mathbb{E} \cdot \mathfrak{O}_{\mathbf{C}} \cdot \mathbb{C}_{\text {Sp }}$. It should be emphasized again that due to the nature of the localic product (frame coproduct) the isomorphisms $\mathbb{C}, \mathbb{E}$ and the embedding $\mathfrak{O}_{\mathbf{E}}$ are somewhat deeper than meets the eye.

\section{LOCALIC GROUPS}

5.1. A group in a category $\mathcal{C}$ with products is a collection of data $(A, m, i, e)$ with

$$
m: A \times A \rightarrow A, \quad i: A \rightarrow A, \quad e: T \rightarrow A \text { morphisms in } \mathcal{C}
$$

such that

$$
\begin{aligned}
& m(m \times \mathrm{id})=m(\mathrm{id} \times m), \\
& m(e \times \mathrm{id})=m(\mathrm{id} \times e)=\mathrm{id}, \quad \text { and } \\
& m(i \times \mathrm{id}) \Delta=m(\mathrm{id} \times i) \Delta=e \cdot t_{A} .
\end{aligned}
$$

( $T=A^{0}$ is the empty product, that is, the terminal object of $\mathcal{C}$, $t_{X}: X \rightarrow T$ is the unique morphism, $\Delta$ is the diagonal morphism). If $(A, m, i, e),\left(A^{\prime}, m^{\prime}, i^{\prime}, e^{\prime}\right)$ are groups in $\mathcal{C}$, the homomorphism $f$ : $(A, m, i, e) \rightarrow\left(A^{\prime}, m^{\prime}, i^{\prime}, e^{\prime}\right)$ is a morphism $f: A \rightarrow A^{\prime}$ such that

$$
f m=m^{\prime}(f \times f), \quad f i=i^{\prime} f \quad \text { and } \quad f e=e^{\prime} .
$$

In particular we will be interested in groups in the category Loc; for convenience we will use the frame language, so that we will consider 
the cogroups in Frm, that is, systems $(L, \mu, \iota, \varepsilon)$ where

$$
\mu: L \rightarrow L \oplus L, \quad \gamma: L \rightarrow L, \quad \varepsilon: L \rightarrow \mathbf{2}=\{0,1\}
$$

are frame homomorphisms such that

$$
\begin{aligned}
& (\mu \oplus \mathrm{id}) \mu=(\mathrm{id} \oplus \mu) \mu, \\
& (\varepsilon \oplus \mathrm{id}) \mu=(\mathrm{id} \oplus \varepsilon) \mu=\mathrm{id}, \quad \text { and } \\
& \nabla(\gamma \oplus \mathrm{id}) \mu=\nabla(\mathrm{id} \oplus \gamma) \mu=\sigma_{L} \varepsilon
\end{aligned}
$$

where $\sigma_{L}: \mathbf{2} \rightarrow L$ sends 0 to 0 and 1 to 1 , and $\nabla$ is the codiagonal $L \oplus L \rightarrow L$, and the homomorphisms (we will call them $L G$ homomorphisms $h:(L, \mu, \iota, \varepsilon) \rightarrow\left(L^{\prime}, \mu^{\prime}, \iota^{\prime}, \varepsilon^{\prime}\right)$ will be frame homomorphisms $h: L \rightarrow L^{\prime}$ such that

$$
\mu^{\prime} h=(h \oplus h) \mu, \quad \gamma^{\prime} h=h \gamma \quad \text { and } \quad \varepsilon^{\prime} h=\varepsilon
$$

The dual to the resulting category, the category of localic groups will be denoted by

\section{LocGr.}

5.2. Localic groups and topological groups. A topological group $(X, m, i, e)$ cannot be always viewed as a localic one: the target $\Omega(X \times X)$ of $\Omega(m)$ cannot be always naturally replaced by $\Omega(X) \oplus$ $\Omega(X)$. More precisely, consider the diagram

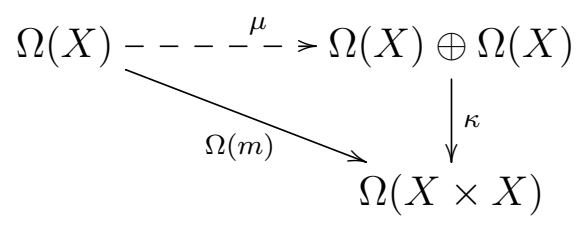

where $\kappa$ is defined by $\kappa \cdot \iota_{i}=\Omega\left(p_{i}\right), p_{i}$ the projections. Now $\kappa$ is always dense (which, for regular frames means it is monomorphic, although it is not typically one-to-one). If $\Omega(m)$ can be lifted to the $\mu$ indicated by the dashed arrow, we have associated with $(X, m, i, e)$ a localic group $(\Omega(X), \mu, \Omega(i), \Omega(e))$. For some important cases (locally compact $X$, complete metric $X$ and others), $\kappa$ is an isomorphism and we have the desired localic group trivially. It is an open problem whether there exists a case of a non-trivial lifting $\mu$ (that is, one with non-isomorphic $\kappa$; this was named by John Isbell as one of the hardest problems of the theory). On the other hand, there is a lot of topological groups that cannot be made to localic ones. For instance, by the Closed Subgroup Theorem ([12], see also [14]) this holds for any topological subgroup that is not closed. 
5.3. It is well known (see e.g. [16]) that the counterpart of each identity that can be deduced in a classical equational class (variety) of algebras holds in the associated category of algebras in $\mathcal{C}$. This spares us a lot of tedious computations in deriving the following identities $\left(\tau: L \oplus L \rightarrow L \oplus L\right.$ is the homomorphism defined by $\tau \iota_{i}=\iota_{3-1}$, $i=1,2)$ :

(5.3.1) $\gamma \gamma=\mathrm{id}\left(\right.$ corresponding to $\left.\left(x^{-1}\right)^{-1}=x\right)$,

(5.3.2) $\varepsilon \gamma=\varepsilon$ (corresponding to $\left.e^{-1}=e\right)$,

(5.3.3) $(\gamma \oplus \gamma) \mu=\tau \mu \gamma$ (corresponding to $\left.(x y)^{-1}=y^{-1} x^{-1}\right)$,

(5.3.4) and the fact that $\alpha=(\mathrm{id} \oplus \nabla)(\mu \oplus \gamma)$ satisfies $\alpha \alpha=\mathrm{id}$ and $\alpha \iota_{1}=\mu\left(\alpha\right.$ corresponds to the mapping $\left.(x, y) \mapsto\left(x y, y^{-1}\right)\right)$.

5.4. Monotone maps $f:(X, \leq) \rightarrow(Y, \leq), g:(Y, \leq) \rightarrow(X, \leq)$, viewed as functors between (thin) categories, are adjoint ( $f$ is the left adjoint and $g$ is the right one) if

$$
f(x) \leq y \quad \text { iff } \quad x \leq g(y)
$$

(in the context of posets one speaks of a Galois adjunction). We will denote the left adjoint $f$ of $g$ by

$$
g_{\#} \cdot
$$

In particular, if $X, Y$ are complete lattices $f$ is a left adjoint (resp. $g$ is a right adjoint) iff it preserves all suprema (resp. infima). Thus in particular each frame homomorphism has a right adjoint. However, we also have

5.4.1. Proposition. (a) The multiplication $\mu$ in a localic group has a left adjoint, and

(b) also $\mu \oplus$ id and id $\oplus \mu$ have left adjoints and there holds

$$
(\mu \oplus \mathrm{id})_{\#}(a \oplus b)=\mu_{\#}(a) \oplus b \quad \text { and } \quad(\mathrm{id} \oplus \mu)_{\#}(a \oplus b)=a \oplus \mu_{\#}(b) .
$$

Proof. (a) Recall (5.3.4). The isomorphism $\alpha$ is its own adjoint, and $\iota_{1}$ has, as it is easy to check, the left adjoint

$$
\left(\iota_{1}\right)_{\#}(u)=\bigvee\{x \mid \exists y \neq 0, x \oplus y \leq u\} .
$$

Thus, we have $\mu_{\#}=\left(\iota_{1}\right)_{\#} \alpha$.

(b) It is easy to check that if $E \in L \oplus L$ is saturated then the union $\bigcup\{\downarrow(\mu(x), y) \mid x \oplus y \leq E\}$ is saturated (recall 1.3) so that

$$
(\mu \oplus \mathrm{id})(E)=\bigcup\{\downarrow(\mu(x), y) \mid x \oplus y \leq E\} .
$$


Hence

$$
\begin{array}{ll} 
& F \leq(\mu \oplus \mathrm{id})(E) \\
\text { iff } \quad \forall a \oplus b \leq F, \quad(a, b) \in \bigcup\{\downarrow(\mu(x), y) \mid x \oplus y \leq E\} \\
\text { iff } \forall a \oplus b \leq F \quad \exists x \oplus y \leq E, a \leq \mu(x) \text { and } b \leq y \\
\text { iff } \quad \forall a \oplus b \leq F \quad \exists x \oplus y \leq E, \mu_{\#}(a) \leq x \text { and } b \leq y \\
\text { iff } \quad \forall a \oplus b \leq F \quad \mu_{\#}(a) \oplus b \leq E \\
\text { iff } \quad \varphi(F)=\bigvee\left\{a \oplus b \mid \mu_{\#}(a) \oplus b \leq E\right\} \leq E .
\end{array}
$$

In particular $(\mu \oplus \mathrm{id})_{\#}(a \oplus b)=\varphi(a \oplus b)=\mu_{\#}(a) \oplus b$.

Note. The first statement is a part of Johnstone's stronger observation ([14]) that $\mu$ is an open homomorphism (open homomorphisms are counterparts of open continuous maps, and are characterized as the Heyting homomorphisms that have left adjoint - see [15]; in the regular case the preservation of the Heyting operation follows from the specific behaviour of congruence in regular frames, see $[13,22])$.

\section{The SEMigroup OF OPEN PARTS}

The algebra $\left(L, *,(-)^{-1}\right)$ to be introduced is a counterpart of the semigroup (with involution) of open subsets of a topological group, with the operations

$$
U V=\{u v \mid u \in U, v \in V\}, \quad U^{-1}=\left\{u^{-1} \mid u \in U\right\} .
$$

It appeared in passing in [4] with the proofs of the properties just hinted. Here we will be more explicit. We will use it for a treatment of the cover uniformities of a localic group, somewhat more elegant than the original procedures in [12].

6.1. Define a (classical) binary operation $*$ and a unary operation $(-)^{-1}$ on $L$ by setting

$$
x * y=\mu_{\#}(x \oplus y), \quad x^{-1}=\gamma(x) .
$$

6.2. Since $\iota_{1}$ is one-one so is $\mu=\alpha \iota_{1}$ (recall (5.3.4)) and we easily infer that

$$
\mu \mu_{\#} \geq \mathrm{id} \text { and } \mu_{\#} \mu=\mathrm{id} \text { and in particular } \mu_{\#}(0)=0 .
$$

6.2.1. Each localic group has at least one point, namely $\varepsilon: L \rightarrow \mathbf{2}$. We will write

$$
N=N_{L}=\{a \mid \varepsilon(a)=1\}
$$


for the set of (representatives of) the neighbourhoods of the unit.

We obviously have

$$
a, b \in N \Rightarrow a \wedge b \in N \quad \text { and } \quad a \in N \Rightarrow a^{-1}=\gamma(a) \in N \text {. }
$$

6.2.2. Proposition. (1) If $x^{\prime} \leq x$ and $y^{\prime} \leq y$ then $x^{\prime} * y^{\prime} \leq x * y$.

(2) If $x=0$ or $y=0$ then $x * y=0$.

(3) The operation $*$ is associative.

(4) If $y \in N$ then $x * y \geq x$ and $y * x \geq x$.

(5) If $x \wedge y \neq 0$ then $x * y^{-1} \in N$.

(6) $(x * y)^{-1}=y^{-1} * x^{-1}$.

(7) If $x \in N$ then $x^{-1} \in N$.

Proof. (1) and (2) are trivial.

(3) Using 5.4.1 we obtain

$$
\begin{aligned}
a *(b * c)=\mu_{\#}\left(a \oplus \mu_{\#}(b \oplus c)\right) & =\mu_{\#}(\mathrm{id} \oplus \mu)_{\#}(a \oplus b \oplus c)= \\
=((\mathrm{id} \oplus \mu) \mu)_{\#}(a \oplus b \oplus c) & =((\mu \oplus \mathrm{id}) \mu)_{\#}(a \oplus b \oplus c)= \\
=\mu_{\#}(\mu \oplus \mathrm{id})_{\#}(a \oplus b \oplus c) & =\mu_{\#}\left(\mu_{\#}(a \oplus b) \oplus c\right)=(a * b) * c .
\end{aligned}
$$

(4) Applying id $\oplus \varepsilon$ on both sides of $\mu \mu_{\#}(x \oplus y) \geq x \oplus y$ we obtain

$$
x * y \geq(\mathrm{id} \oplus \varepsilon)(x \oplus y)=x \oplus \varepsilon(y)=x \wedge \varepsilon(y) .
$$

(5) Since we have

$$
\begin{aligned}
\sigma \varepsilon\left(x * y^{-1}\right) & =\nabla(\mathrm{id} \oplus \gamma) \mu \mu_{\#}(\mathrm{id} \oplus \gamma)(x \oplus y) \geq \\
& \geq \nabla(\mathrm{id} \oplus \gamma)(\mathrm{id} \oplus \gamma)(x \oplus y)=x \wedge y \neq 0,
\end{aligned}
$$

$\varepsilon\left(x * y^{-1}\right)$ cannot be 0 .

(6) Since $\tau_{\#}=\tau$ and $\gamma_{\#}=\gamma$, we obtain from (5.3.3) that

$$
\mu_{\#}(\gamma \oplus \gamma)=\gamma \mu_{\#} \tau
$$

and as $\tau(x \oplus y)=y \oplus x$ we conclude

$$
x^{-1} * y^{-1}=\mu_{\#}(\gamma(x) \oplus \gamma(y))=\gamma \mu_{\#}(y \oplus x)=(y * x)^{-1} .
$$

(7) follows from (5.3.2).

6.3.1. Lemma. If $c * b \leq a, u * u^{-1} \leq c$ and $u \wedge b \neq 0$ then $u \leq a$.

Proof. We have $c \oplus b \leq \mu(a)$ and $u \oplus \gamma(u) \leq \mu(c)$ so that $u \oplus \gamma(u) \oplus b \leq \mu(c) \oplus b=(\mu \oplus \mathrm{id})(c \oplus b) \leq(\mu \oplus \mathrm{id}) \mu(a)=(\operatorname{id} \oplus \mu) \mu(a)$. Applying id $\oplus \nabla(\gamma \oplus$ id) we obtain on the leftmost side $u \oplus(u \wedge b)$ (as $\nabla(x \oplus y)=x \wedge y)$ and on the rightmost one

$$
\begin{aligned}
& (\mathrm{id} \oplus(\nabla(\gamma \oplus \mathrm{id}) \mu) \mu(a)=(\mathrm{id} \oplus \sigma \varepsilon) \mu(a)= \\
& \quad=(\operatorname{id} \oplus \sigma)(\mathrm{id} \oplus \varepsilon) \mu(a)=(\operatorname{id} \oplus \sigma)(a)=a \oplus 1 .
\end{aligned}
$$


Thus $u \oplus(u \wedge b) \leq a \oplus 1$ and since $u \wedge b \neq 0, u \leq a$.

6.3.2. Lemma. For each $a \in N$ there are $b, c \in N$ such that $b * b \leq a$ and $c * c^{-1} \leq a$.

Proof. Any $L$ can be viewed as a coproduct

$$
2 \stackrel{\sigma_{L}}{\longrightarrow} L \stackrel{\operatorname{id}_{L}}{\longleftarrow} L
$$

and since $\sigma_{\mathbf{2}}=\mathrm{id}_{\mathbf{2}}$ we have

$$
\varepsilon=\operatorname{id}_{\mathbf{2}} \oplus \varepsilon: L=\mathbf{2} \oplus L \rightarrow \mathbf{2} \oplus \mathbf{2}=\mathbf{2} .
$$

Hence, $\varepsilon=\left(\operatorname{id}_{\mathbf{2}} \oplus \varepsilon\right)\left(\varepsilon \oplus \operatorname{id}_{L}\right) \mu=(\varepsilon \oplus \varepsilon) \mu$ and we obtain, for $a \in N$,

$1=\varepsilon(a)=\bigvee\{\varepsilon(x) \oplus \varepsilon(y) \mid x \oplus y \leq \mu(a)\}=\bigvee\{\varepsilon(x) \oplus \varepsilon(y) \mid x * y \leq a\}$

so that there are $x, y$ such that $x * y \leq a$ and $\varepsilon(x)=\varepsilon(y)=1$. Set $b=x \wedge y$ and $c=x \wedge \gamma(y)$.

\section{UNIFORMITIES ON LOCALIC GROUPS}

7.1. For an $a \in N$ set

$$
\begin{aligned}
& U(a)=\{x \in L \mid x \oplus \gamma(x) \leq \mu(a)\}=\left\{x \in L \mid x * x^{-1} \leq a\right\}, \\
& V(a)=\{x \in L \mid \gamma(x) \oplus x \leq \mu(a)\}=\left\{x \in L \mid x^{-1} * x \leq a\right\}
\end{aligned}
$$

and consider the systems

$$
\mathcal{U}=\{U \mid U \geq U(a), \varepsilon(a)=1\} \text { and } \mathcal{V}=\{V \mid V \geq V(a) \varepsilon(a)=1\} .
$$

\subsubsection{Proposition. $\mathcal{U}$ and $\mathcal{V}$ are uniformities on $L$.}

Proof. It will be done for $\mathcal{U}$.

I. Each $U(a)$ is a cover. We have

$$
U(a)=\{x \wedge y \mid x \oplus y \leq(\mathrm{id} \oplus \gamma) \mu(a)\} .
$$

(Indeed, if $x \oplus \gamma(x) \leq \mu(a)$ then $x \oplus x \leq(\operatorname{id} \oplus \gamma)(x \oplus \gamma(x)) \leq(\operatorname{id} \oplus \gamma) \mu(a)$. On the other hand, if $x \oplus y \leq(\mathrm{id} \oplus \gamma) \mu(a)$ then $(x \wedge y) \oplus \gamma(x \wedge y) \leq$ $(\mathrm{id} \oplus \gamma)(x \oplus y) \leq(\mathrm{id} \oplus \gamma)(\mathrm{id} \oplus \gamma) \mu(a)=\mu(a)$.)

Thus,

$$
\begin{aligned}
& \bigvee U(a)=\bigvee\{x \wedge y \mid x \oplus y \leq(\operatorname{id} \oplus \gamma) \mu(a)\}= \\
& =\bigvee\{\nabla(x \oplus y) \mid x \oplus y \leq(\operatorname{id} \oplus \gamma) \mu(a)\}= \\
& =\nabla \bigvee\{x \oplus y \mid x \oplus y \leq(\operatorname{id} \oplus \gamma) \mu(a)\}=\nabla(\operatorname{id} \oplus \gamma) \mu(a)=\sigma \varepsilon(a)=1
\end{aligned}
$$


II. The system $\mathcal{U}$ is admissible. By 6.3.1, if $c * b \leq a$ then $U(c) b \leq a$. We have $a=(\varepsilon \oplus \mathrm{id}) \mu(a)=\bigvee\{\varepsilon(c) \oplus b \mid c \oplus b \leq \mu(a)\}=\bigvee\{b \mid u * b \leq$ $a, c \in N\} \leq \bigvee\{b \mid U(c) b \leq a, c \in N\}$.

III. Trivially $U(a \wedge b) \leq U(a) \wedge U(b)$.

IV. For $a \in N$ choose, by 6.3 .2 , a $b \in N$ such that $b * b * b^{-1} * b^{-1} \leq a$. We will show that $U(b) U(b) \leq U(a)$.

Fix an $x \in U(b)$ and consider any $u \in U(b)$ such that $u \wedge x \neq 0$. Thus, $x * x^{-1} \leq b$ and $u * u^{-1} \leq b$ and, by 6.2.2, $(u \wedge x)^{-1} *(u \wedge x) \in N$. Thus,

$$
u \leq u *(u \wedge x)^{-1} *(u \wedge x) \leq u * u^{-1} * x \leq b * x
$$

and hence $U(b) x \leq b * x$ and finally, since also $b^{-1} \in N$, again by 6.2.2,

$U(b) x *(U(b) x)^{-1} \leq b * x * x^{-1} * b^{-1} \leq b * b * b^{-1} \leq b * b * b^{-1} * b^{-1} \leq a$ and $U(b) x \in U(a)$.

7.1.2. The uniformity $\mathcal{U}$ (resp. $\mathcal{V}$ ) is called the left uniformity (resp. right uniformity) on the localic group.

7.1.3. Note. The cover uniformities just defined remind us of the intuition in classical topological groups where one can think of the basic covers as obtained by taking a neighbourhood of the unit and shifting it by the obvious homeomorphisms $x \mapsto a x$ to all the other points $a$. It should be noted, however, that a localic group does not have to be spatial; indeed it may be arbitrarily large with the unit being the only point (see [12]).

7.2. In view of the naturalness of the uniformities (and recalling the classical facts) we may well ask whether the correspondences $L \mapsto$ $(L, \mathcal{U}(L))$ resp. $L \mapsto(L, \mathcal{V}(L)), L \in \mathbf{L o c G r}$, can be extended to functors

\section{LocGr $\rightarrow$ Uni $_{\text {Coc }}$,}

that is, whether each LG-homomorphism $h: L \rightarrow M$ is a uniform homomorphism $(L, \mathcal{U}(L)) \rightarrow(M, \mathcal{U}(M))$. Proving this does not seem to be easy. However, we can present an extremely simple and straightforward proof if we do it for the entourages instead.

7.3. For an $a \in N$ set

$$
E(a)=(\mathrm{id} \oplus \gamma) \mu(a) \quad \text { and } \quad F(a)=(\gamma \oplus \mathrm{id}) \mu(a) ;
$$

hence

$$
E(a)=\bigvee\{x \oplus y \mid x \oplus y \leq(\mathrm{id} \oplus \gamma) \mu(a)\}=\bigvee\{x \oplus y \mid x * y \leq a\},
$$

and similarly for $F(a)$. 
7.3.1. Observation. $E(a)$ and $F(a)$ are entourages.

Proof. For $x \in U(a)$ we have $x \oplus \gamma(x) \leq \mu(a)$. Hence

$$
\bigvee\{x \mid x \oplus x \leq E(a)\} \geq \bigvee U(a)=1
$$

since $U(a)$ is a cover, as we already know.

7.3.2. Denote by $\mathcal{E}^{\prime}$ resp. $\mathcal{F}^{\prime}$ the system of entourages $\{E(a) \mid a \in$ $N\}$ resp. $\{F(a) \mid a \in N\}$, and set

$$
\begin{aligned}
& \mathcal{E}=\left\{E \mid E \text { entourage, } E \geq E(a) \in \mathcal{E}^{\prime}\right\}, \\
& \mathcal{F}=\left\{E \mid E \text { entourage, } E \geq F(a) \in \mathcal{F}^{\prime}\right\} .
\end{aligned}
$$

7.3.3. Proposition. The systems $\mathcal{E}$ and $\mathcal{F}$ are e-uniformities and we have, in the notation of 7.1 and $4.4, \mathcal{E}=\mathcal{E}_{\mathcal{U}}$ and $\mathcal{F}=\mathcal{E}_{\mathcal{V}}$.

Proof. We will show that $\mathcal{E}=\left\{E \mid E\right.$ entourage, $\left.E \geq E(a) \in \mathcal{E}^{\prime}\right\}=$ $\mathcal{E}_{\mathcal{U}}=\left\{E \mid E\right.$ entourage, $\left.E \geq E_{U(a)}, a \in N\right\}$.

We have $E_{U(a)}(=\bigvee\{x \oplus x \mid x \oplus \gamma(x) \leq \mu(a)\}) \leq E(a)$.

To obtain an estimate from the other side, choose by $6.3 .2 b, c \in N$ such that $b * b^{-1} \leq c$ and $c * c^{-1} \leq a$. Let $x \oplus y \leq E(b)$. We can assume $x \oplus y \neq 0$, hence $x \neq 0 \neq y$. First, as $y \neq 0$, we have by 6.2 .2 ((4), (5) and $(7)$ ),

$$
x * x^{-1} \leq x * y^{-1} * y * x^{-1} \leq b * b^{-1} \leq c \quad \text { and } \quad x * y^{-1} \leq b * b^{-1} \leq c
$$

and hence $(x, x),(x, y) \in E(c)$ and since $E(c)$ is saturated (recall 1.3) we have, for $z=x \vee y$,

$$
(x, z) \in E(c) \text {, that is, } \quad x * z^{-1} \leq c .
$$

Now $\left(x * z^{-1}\right) *\left(x * z^{-1}\right)^{-1} \leq c * c^{-1} \leq a$, hence $\left(x * z^{-1}\right) \oplus\left(x * z^{-1}\right) \leq E_{U(a)}$ and $\left(x * z^{-1}\right) *\left(x * z^{-1}\right)^{-1} \leq \mu\left(E_{U(a)}\right)$. Since $x \wedge z \neq 0$ we have $\left(x * z^{-1}\right)^{-1} \in N$ by 6.2.2(5), and by 6.2.2(4) we obtain

$$
x * z^{-1} \leq x * z^{-1} *\left(x * z^{-1}\right)^{-1} \leq \mu\left(E_{U(a)}\right)
$$

so that $x \oplus y \leq x \oplus z \leq E_{U(a)}$. Thus, $E(b) \leq E_{U(a)}$.

Remarks. Note that we did not have to prove that $\mathcal{E}$ is a uniformity. It followed from the fact that $\mathcal{E}_{\mathcal{U}}$ is one.

7.4. Theorem. Each LG-homomorphism $h:\left(L, \mu_{L}, \gamma_{L}, \varepsilon_{L}\right) \rightarrow$ $\left(M, \mu_{M}, \gamma_{M}, \varepsilon_{M}\right)$ is uniform with respect to both the left and the right uniformities. Thus we have identity carried functors

$$
\begin{aligned}
& \left(L \mapsto(L, \mathcal{E}(L)) \text { resp. }(L, \mathcal{F}(L)): \text { LocGr } \rightarrow \text { Uni }_{\mathbf{E}} \mathbf{L o c} \quad\right. \text { and } \\
& \left(L \mapsto(L, \mathcal{U}(L)) \text { resp. }(L, \mathcal{V}(L)): \mathbf{L o c G r} \rightarrow \mathbf{U n i}_{\mathbf{C}}\right. \text { Loc. }
\end{aligned}
$$


Proof. We will prove it for the left uniformity. By 7.3.2 and 4.4.2 we can choose whether we will prove it for the $\mathcal{U}$ or for the entourageuniformity $\mathcal{E}$. We will do it for the latter. By (5.1.1) we have

$$
\begin{aligned}
& (h \oplus h)(E(a))=(h \oplus h)\left(\operatorname{id}_{L} \oplus \gamma\right) \mu_{L}(a)= \\
& \quad=\left(\operatorname{id}_{M} \oplus \gamma_{M}\right)(h \oplus h) \mu_{L}(a)=\left(\operatorname{id}_{M} \oplus \gamma_{M}\right) \mu_{M}(h(a))=E(h(a))
\end{aligned}
$$

(since $\varepsilon_{M} h=\varepsilon_{L}, h(a) \in N_{M}$, and $E(h(a))$ makes sense).

\section{REFERENCES}

[1] B. Banaschewski, H.S. Hong and A. Pultr, On the completion of nearness frames, Quaestiones Math. 21 (1998), 19-37.

[2] B. Banaschewski and A. Pultr, Cauchy points of uniform and nearness frames, Quaestiones Math. 19 (1996), 101-127.

[3] B. Banaschewski and A. Pultr, On Cauchy homomorphisms of nearness frames, Math. Nachr. 183 (1997), 5-18.

[4] B. Banaschewski and J. C. C. Vermeulen, On the completeness of localic groups, Comment. Math. Univ. Carolinae 40 (1999), 293-307.

[5] C. H. Dowker and D. Strauss, Sums in the category of frames, Houston J. Math. 3 (1977), 7-15.

[6] M. J. Ferreira and J. Picado, The Galois approach to uniform structures, Quaest. Math. 28 (2005), 355-373.

[7] P. Fletcher and W. Hunsaker, Entourage uniformities for frames, Monatsh. Math. 112 (1991), 271-279.

[8] P. Fletcher, W. Hunsaker and W. Lindgren, Characterizations of frame uniformities, Quaestiones Math. 16 (1993), 371-383.

[9] J. L. Frith, Structured Frames, Ph.D. Thesis, University of Cape Town, 1987.

[10] J. R. Isbell, Uniform spaces, Math. Surveys, vol. 12, Amer. Math. Soc., 1964.

[11] J. R. Isbell, Atomless parts of spaces, Math. Scand. 31 (1972), 5-32.

[12] J. R. Isbell, I. Kř́žz, A. Pultr and J. Rosický, Remarks on localic groups, in Categorical Algebra and its Applications (Proc. Int. Conf. Louvain-La-Neuve 1987, ed. by F. Borceux), Lecture Notes in Math. 1348, pp. 154-172.

[13] P. T. Johnstone, Stone Spaces, Cambridge Studies in Advanced Math. No 3, Cambridge University Press, Cambridge, 1983.

[14] P. T. Johnstone, A simple proof that localic groups are closed, Cahiers Topologie Géom. Différentielle Catég. 29 (1988), 157-161.

[15] A. Joyal and M. Tierney, An extension of the Galois theory of Grothendieck, Mem. Amer. Math. Soc. 51 (1984), no. 309.

[16] A. Kock, Limit Monads in Categories, Ph.D. Thesis, University of Chicago, 1967.

[17] S. MacLane, Categories for the Working Mathematician, Springer-Verlag, New York, 1971.

[18] J. Picado, Weil entourages in Pointfree Topology, Ph.D. Thesis, University of Coimbra, 1995.

[19] J. Picado, Weil uniformities for frames, Comment. Math. Univ. Carolinae 36 (1995), 357-370. 
[20] J. Picado, Structured frames by Weil entourages, Appl. Categ. Structures 8 (2000), 351-366.

[21] A. Pultr, Pointless uniformities I,II, Comment. Math. Univ. Carolinae 25 (1984), 91-104, 105-120.

[22] A. Pultr, Frames, Chapter in: Handbook of Algebra, Vol. 3, (ed. by M. Hazewinkel), Elsevier 2003, 791-858.

[23] J. W. Tukey, Convergence and uniformity in topology, Ann. Math. Stud. 2, Princeton University Press, 1940.

[24] A. Weil, Sur les espaces à structure uniforme et sur la topologie générale, Publications de l'Institute Mathématique de l'Université de Strasbourg, Hermann, Paris, 1938.

CMUC, Department of Mathematics, University of Coimbra, 3001454 CoImbra, PORTUGAL

E-mail address: picado@mat.uc.pt

Department of Applied Mathematics and iti, MFF, Charles UniverSITY, PRAHA, CZECH REPUBLIC

E-mail address: pultr@kam.ms.mff.cuni.cz 